\title{
La formación de docentes para la inclusión educativa. Escenarios de justicia social en España y México
}

\author{
Juárez Romero, Claudia Amanda \\ Benemérita Escuela Nacional de Maestros, Ciudad de México,México \\ amandajuarezrom.unam@gmail.com \\ Alcántara Santuario, Armando \\ Universidad Nacional Autónoma de México, Ciudad de México,México. \\ aralsantuario@gmail.com \\ Miñán Espigares, Antonio \\ Universidad de Granada, Granada,España. \\ aminan@ugr.es
}

\section{Resumen}

Para contar con docentes preparados para desarrollar escuelas inclusivas, los sistemas deben preocuparse de su formación. La cuestión de partida es averiguar cuál debe ser el perfil del docente inclusivo. Este perfil ha sido estudiado por la Agencia Europea para el desarrollo de la Educación de alumnado con Necesidades Educativas Especiales (2012), agrupando las competencias en cuatro valores: 1. Valorar la diversidad del alumnado (la diversidad es un recurso y un valor), 2. Apoyar a todo el alumnado (sacar lo mejor de cada uno), 3. Trabajar en equipo, 4. Desarrollo profesional permanente. Algunas de las competencias están relacionadas con: ser capaces de generar un aula democrática, solidaria y participativa, transmitir altas expectativas de progreso en los estudiantes, flexibilizar y adaptar el curriculum e innovar la enseñanza relacionándola con situaciones de la vida. En este trabajo de educación comparada nos planteamos averiguar cómo perciben los futuros docentes el nivel de inclusión que existe en las Escuelas donde realizan prácticas. Para ello se utiliza un cuestionario adaptado a partir del trabajo de Booth y Ainscow (2000). En la valoración que les pedimos se ponen en juego los conocimientos que obtienen en la formación inicial sobre inclusión educativa y su capacidad de comprensión del contexto escolar. La muestra está formada por estudiantes de Magisterio de México y de España. El contraste entre los resultados obtenidos de ambos países nos permitirá extraer conclusiones que orienten las medidas de mejora de la formación de docentes para colaborar con escenarios de justicia social a implementar en los programas de formación de docentes de México y de España. El enfoque correcto, siguiendo a Bolívar (2011) es el de encontrar conocimientos que hagan avanzar y hacer paulatinamente más justas las sociedades reales, en lugar de definir una justicia perfecta y establecer las condiciones que deben cumplir las instituciones.

\section{Abstract}

In order to have teachers prepared for the development of inclusive schools, systems must be committed with their training. The starting point should be the profile of the inclusive teacher. This profile has been studied by the European Agency for the Development of the Education of Students with Special Education Needs (2012), grouping the competences into four values: 1 . To value the diversity of students (diversity is a resource and a value), 2. Supporting all students (get the best out of each one), 3. Working as a team, 4. Permanent professional development. Some of the competences are related to the ability to generate a democratic, solidarity and participatory classroom, transmit high expectations of progress in students, make flexible and adaptthe curriculum and innovate teaching relating it to life situations. In this comparative education work, we consider how future teachers can find the level of inclusion that exists in the schools where practices are carried out. For this, a questionnaire adapted from the work of Booth and Ainscow (2000) is used. In the evaluation that we ask them, the knowledge they got in the initial formation about educational inclusion and their ability to understand the school context are put at stake. The sample is formed by «magisterio» (teacher training) students of Mexico and Spain. The contrast between the results obtained from both countries allows us to draw conclusions that guide the improvement measures of teacher training to collaborate with the social justice scenarios to be implemented in teacher training programs in Mexico and Spain. The correct approach, following Bolívar (2011), is to reach the knowledge that advances and gradually real societies, instead of defining perfect justice and establishing the conditions that institutions must meet.

Palabras clave: educación comparada, diversidad, formación docente, inclusión educativa, justicia social.

Keywords: comparative education, diversity, teacher training, educational inclusion, social justice. 


\section{INTRODUCCIÓN}

La justicia social plantea la necesidad de lograr un reparto equitativo de los bienes sociales. En una sociedad con justicia social los derechos humanos son respetados y las clases y grupos sociales más desfavorecidos cuentan con oportunidades de desarrollar todas sus capacidades. En términos más operativos, la justicia social consiste en un conjunto de políticas que tienen como objetivo reducir la desigualdad por la que atraviesan los grupos más vulnerables de la sociedad.

En las sociedades contemporáneas una educación de calidad para todos es condición necesaria para el logro de la justicia social. En general, la educación es condición necesaria para la inclusión social, así como para tomar decisiones, expresar demandas y construir un proyecto de vida (Tedesco, 2012).

La exigencia de construir sociedades más justas se ha intensificado, tanto por las potencialidades de la injusticia que produce el nuevo capitalismo como por la generalización de las demandas de democracia, de respeto a los derechos humanos y de reconocimiento a las identidades culturales, étnicas, lingüísticas y de género (Tedesco,2012).

Para Dubet (2011), existe una diferencia entre políticas de igualdad de lugares y políticas de igualdad de oportunidades. La primera se relaciona con la estructura que constituye el conjunto de posiciones sociales ocupadas por las personas, que puede ser más o menos injusta según las distancias entre las categorías más altas y las más bajas. En esta perspectiva, las políticas de justicia social procuran reducir las desigualdades de ingresos, de condiciones de vida y de acceso a los servicios que hay entre las diferentes posiciones sociales, sin que se le dé prioridad a la movilidad de las personas. Por el contrario, la segunda se centra en la igualdad de oportunidades y se caracteriza por ofrecer a todos la misma posibilidad de ocupar las mejores posiciones, en función de sus méritos, sin cuestionar el carácter abiertamente jerárquico de la estructura de posiciones.

Para que exista igualdad de oportunidades es preciso que la Educación sea inclusiva, que eduque a todas las personas sin excluir a nadie. Una Educación Inclusiva debe reconocer las diferencias, las singularidades de cada persona, situando en un lugar prioritario el enriquecimiento que se produce como consecuencia de la interacción positiva, educativa, entre personas diferentes. Lamentablemente, la inclusión, no se produce automáticamente, ni a nivel escolar, ni a nivel social. Se requiere por una parte formación adecuada de los docentes para atender las diferencias, según ritmos diferentes, características, capacidades, estilos de aprendizaje o procedencia social. Para que se produzca la inclusión se necesitan cambios de mentalidad, de actitud, sobre todo de los docentes, ya que estas actitudes provienen de visiones o perspectivas antiguas, tradicionales, no fundamentadas, pero que están arraigadas y perpetúan la desigualdad.

La inclusión debe ser considerada como una búsqueda interminable de formas más adecuadas de responder a la diversidad. Se trata de aprender a convivir con la diferencia y de aprender de la diferencia. De este modo la diferencia es un factor más positivo y un estímulo para el aprendizaje de menores y adultos. (Ainscow, 2003, citado en Fernández, 2014). (p. 25)

En México, el Consejo Nacional para el Desarrollo y la Inclusión de las Personas con Discapacidad (CONADIS) y la Secretaría de Educación Pública (SEP), trabajan de manera conjunta para impulsar acciones que fortalezcan la inclusión educativa de las personas con discapacidad. La SEP mediante el Programa para la Inclusión y la Equidad Educativa (PIEE), creado en 2014, la SEP pretende:

contribuir a asegurar una mayor cobertura, inclusión y equidad educativa entre todos los grupos de la población para la construcción de una sociedad más justa mediante normas y apoyos para los servicios educativos públicos, así como el mejoramiento de infraestructura y equipamiento de instituciones de educación básica, media superior y superior, que atienden población en contexto de vulnerabilidad. (SEP-PIEE,2014).

El concepto de inclusión educativa en México, ha evolucionado en los últimos años: solía asociarse con los estudiantes que vivían en situaciones de alta marginación o que tenían necesidades especiales. En la actualidad existe un consenso entre los países de la región latinoamericana para ampliar el concepto y lograr un mayor acceso a una educación de calidad sin discriminación alguna, considerando a niños y niñas con discapacidad y/o con capacidades sobresalientes; poblaciones indígenas; poblaciones rurales; migrantes; y estudiantes que han abandonado el sistema educativo. 
En España, la situación actual viene determinada por la Ley Orgánica General del Sistema Educativo, de 2013, (LOE-LOMCE), consecuencia de una evolución legal, educativa y social en estas últimas dos décadas, en todas las Comunidades Autónomas españolas, así como por la asunción y ratificación de normas internacionales en favor de la inclusión y de las personas con discapacidad, tales como la Declaración de Incheon, República de Corea, 21 de mayo de 2015, por la que se pone de manifiesto la necesidad de «realizar los cambios oportunos en las políticas de educación y a centrar nuestros esfuerzos en los más desfavorecidos, especialmente aquellos con discapacidad, para velar porque nadie se quede atrás».

Así por ejemplo en la comunidad andaluza, se establece que:

Un sistema educativo inclusivo debe garantizar la equidad en el acceso, en la permanencia y en la búsqueda del mayor éxito escolar de todo el alumnado. Para ello, es preciso contar con un sistema de prevención, detección e identificación de las necesidades educativas que el alumnado pudiese presentar a lo largo de su escolarización para poder adoptar la respuesta educativa que cada caso requiera. (Instrucciones de 8 de marzo de 2017, Junta de Andalucía)

La Confederación Española de Organizaciones a favor de las Personas con Discapacidad Intelectual FEAPS, en 2009, realizó un estudio sobre la situación española de la inclusión educativa, de la que podemos extraer las siguientes conclusiones:

- Existe cierta apatía en los profesionales que conviene reavivar porque la inclusión educativa requiere impulso continuo.

- La enseñanza, en general, continúa orientada hacia la homogeneidad en clase, como si no hubiera problemas de rendimiento ni diversidad. Es preciso un cambio que actualice al profesorado y permita una enseñanza orientada a la diversidad y esté basada en el diseño universal de aprendizaje.

- Debe promoverse una dotación de recursos acorde con las ideas de inclusión: infraestructuras que permitan agrupaciones flexibles, número de profesores según las necesidades del grupo, adaptaciones de materiales, etc.

En muchos países, se han diseñado e implementado políticas educativas en aras de consolidar la igualdad de oportunidades, la equidad y la inclusión para la población infantil y juvenil, como consecuencia de los efectos de la globalización y el neoliberalismo en la educación a partir del inicio del sigloXXI.

La UNESCO, considera la inclusión como un proceso que permite tener debidamente en cuenta la diversidad de las necesidades de todos los niños, jóvenes y adultos a través de una mayor participación en el aprendizaje, las actividades culturales y comunitarias, así como reducir la exclusión de la esfera de la enseñanza y dentro de ésta, y en último término acabar con ella. (UNESCO, 2009, p. 9).

Es importante mencionar que los Organismos Internacionales han orientado también el diseño de políticas de inclusión y han impulsado acciones educativas en diferentes países como es el caso de Latinoamérica yMéxico.

La educación inclusiva supone transformar la sociedad con la participación de autoridades, instituciones, padres de familia, docentes y estudiantes para favorecer la construcción de las condiciones para la justicia social y la equidad.

A partir de los compromisos establecidos en la Conferencia Internacional de Educación para todos (ETP) llevada a cabo en Jomtiem en 1990 se hizo latente la necesidad de establecer como prioridad el derecho a la educación y la satisfacción de necesidades básicas de aprendizaje.

En México, la inclusión es uno de los objetivos primordiales de las últimas Reformas Educativas tanto en educación básica como para la formación de docentes.

Los cambios curriculares implementados están sustentados en el Marco Jurídico Mexicano, en este sentido, Alcántara y Navarrete (2013), reconocen la apertura que en los últimos años ha tenido el gobierno, en materia legislativa, en torno al tema de la inclusión e igualdad de derechos para los habitantes del pueblo mexicano. Algunos casos en concreto son la creación de leyes para prevenir y eliminar la discriminación, para la inclusión de las personas con discapacidad, para la igualdad entre mujeres y hombres; así como la creación de sistemas, consejos, programas institucionales, 
acciones educativas, fallos judiciales, mecanismos de protección, encuestas, campañas, estudios y muchas otras acciones para prevenir y eliminar la discriminación. Pero, tal como afirma el presidente del Conapred, Ricardo Bucio (2012, citado por Alcántara y Navarrete, 2013), no se ha logrado revertir el sistema de desigualdad de trato y de distinción en el ejercicio de derechos y libertades. Es probable que esto se deba ala fragmentación de los esfuerzos así como a la falta de coherencia y de sistematicidad con que las distintas instituciones responsables han conducido la política antidiscriminatoria (Alcántara y Navarrete, 2013, p. 233).

Los cambios curriculares implementados en los planes y programas para la formación de profesores de educación básica desde 1997 integraron el tema de necesidades educativas especiales, en las cinco Licenciaturas: Educación Preescolar, Primaria, Secundaria, Educación Física y Educación Especial. La Reforma Curricular llevada a cabo en el 2012 integró un espacio curricular sobre «Atención a la diversidad».

En México, la formación de docentes de Educación Básica es responsabilidad de las Escuelas Normales, instituciones que se insertaron al nivel terciario en la década de los ochenta. Es importante mencionar que, las últimas reformas educativas han atendido aspectos establecidos de la agenda internacional y nacional como lo son la atención a la diversidad e inclusión educativa.

A partir de 1992, con el Acuerdo Nacional para la Modernización de la Educación Básica, se ha promovido la integración educativa de los alumnos con discapacidad a las escuelas de educación básica regular, así como la transformación de los servicios de educación especial (USAER, CAM, CAPEP); desde esa fecha se han establecido en nuestro país diferentes acciones para la integración e inclusión educativa, que tienen como eje rector los apoyos que se le pueden proporcionar al alumno al interior de la escuela para la satisfacción de sus necesidades de aprendizaje. "Se dejó de considerar que el niño tiene un problema que debe ser resuelto y, en cambio, se asumió que tiene algunas necesidades que la escuela no pueden satisfacer con los recursos que utiliza habitualmente». (SEP: 2002, p.13).

Para la implementación de la Reforma Curricular 2012 se intentó resolver algunas problemáticas, tales como los programas de estudio rezagados y desfase con reforma de Educación Básica.

Los documentos normativos que sustentaron el nuevo esquema de formación de docentes son: Art $3^{\circ}$ de la Constitución Política de los Estados Unidos Mexicanos, Ley Orgánica de la Administración Pública Federal, Ley General de Educación, Reglamento Interior de la SEP, Plan Desarrollo Nacional 2006-2012.

En la Reforma Curricular de la Educación Normal 2012 en las Licenciaturas de Educación Preescolar, Primaria, Preescolar Intercultural Bilingüe podemos destacar algunas características:

- Se incorporan en la malla curricular cursos optativos de acuerdo con sus intereses y necesidades, así como a los proyectos y posibilidades institucionales.

- Se promueve la participación de los colegiados de profesores de las Escuelas Normales ante las autoridades educativas locales, para proponer contenidos regionales que habrán de incluirse en la formación de los estudiantes.

- Se fortalece la formación disciplinar en las áreas de conocimiento que se relacionan con la Educación Básica (Trayecto de Prácticas).

El espacio curricular atención a la diversidad del Plan de Estudios 2012 de la Licenciatura en Educación Primaria se encuentra ubicado en el campo formativo Psicopedagógico y se cursa en el quinto semestre.

A través de las Unidades de aprendizaje se forma a los futuros docentes para la apropiación de una perspectiva social en el tema de la diversidad, de tal manera, que los estudiantes durante las jornadas de práctica en contextos reales puedan crear las condiciones idóneas para el aprendizaje hacia la conformación de aulas incluyentes para favorecer la equidad de género y condición social hacia la inclusión educativa y social de los alumnos con capacidades diferentes, discapacidades, situación vulnerable o de riesgo, y atendiendo también el tema de diálogo intercultural.

Julieta Zacarías menciona que todo proyecto escolar incluyente, en la conformación y fortalecimiento de la cultura escolar, implica el análisis de los siguientes apartados íntimamente relacionados:

a. la cultura escolar y los métodos cooperativos de enseñanza.

b. las relaciones de colaboración y apoyo en el interior de la escuela.

c. la relación de esta con factores externos y su vinculación con la comunidad. (Zacarías, 2006). 
El programa promueve el desarrollo de competencias genéricas y profesionales que le permiten al estudiante normalista tener una visión que propicie y regule espacios de aprendizaje incluyentes para los alumnos de educación primaria con la finalidad de promover la convivencia, los valores como el respeto, la tolerancia, entre otros. Las fortalezas de este espacio curricular son: a) parte de una situación problemática, b) se busca educar desde la igualdad, desde el respeto al otro, y c) retoma la transversalidad (SEP, 2012,2017)

Los docentes deben comprender las tres razones de la inclusión, que constituyen los tres escenarios de justicia social, que quedan resumidos de la siguiente forma:

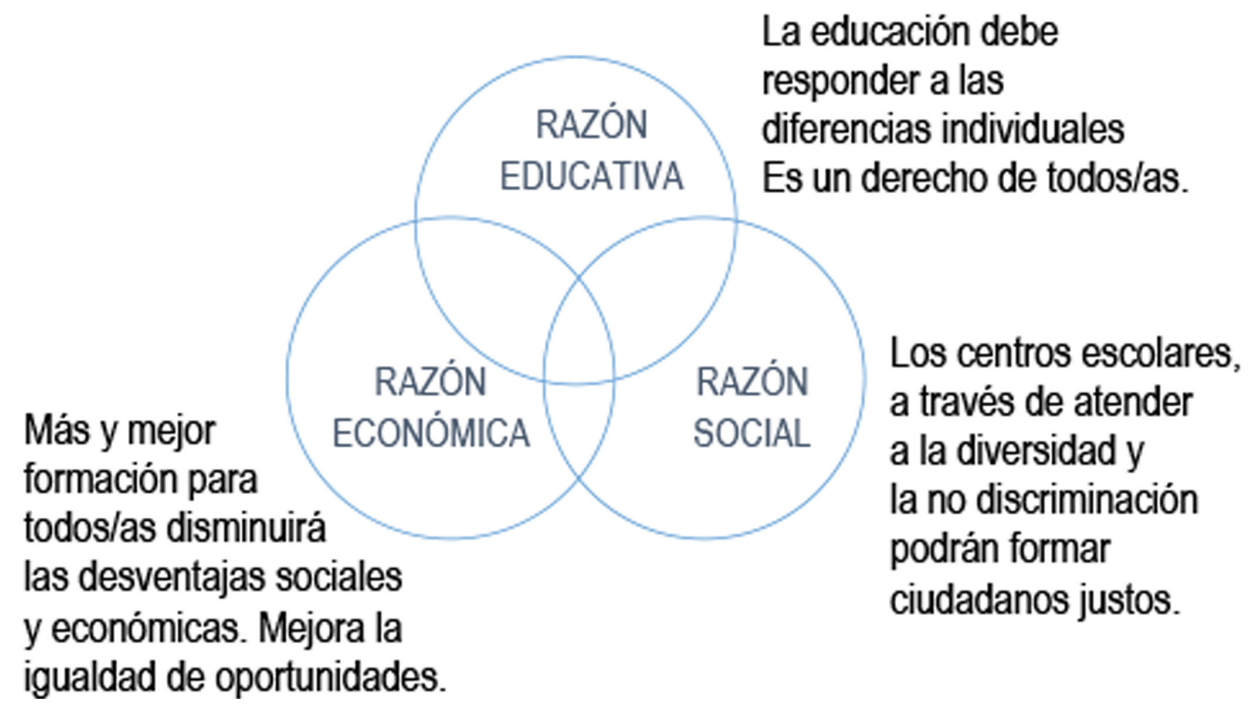

Figura 1: Razones de la Inclusión.

En España, el escenario de la Formación de docentes queda marcado con la implantación del Espacio Europeo de Educación Superior en el año 2010. La Formación Inicial se estructura en dos especialidades básicas, Magisterio Educación Infantil y Educación Primaria. También se imparten en las Facultades de Educación, las especialidades de Educación Social y de Pedagogía. En las cuatro especialidades existen materias troncales y optativas relacionadas con la Atención a la Diversidad. Igual que con la formación para profesorado de secundaria.

Con esta formación se pretende que adquieran los conocimientos básicos sobre educación inclusiva, que sean profesionales críticos, que el modo en el que se concretan las distintas posibilidades del Sistema Educativo actual, a la hora de ofrecer respuestas educativas adecuadas a las distintas necesidades del alumnado.

Creemos que los escenarios de justicia social se deben fundamentar en la definición de unas competencias específicas que debe adquirir el profesorado. Según Cotrina y García (2013) algunas competencias importantes son: Capacidad de generar aulas democráticas, de manejar un aula diferenciada, donde se valorizan las diferencias, de innovar, etc.

En este trabajo nos planteamos qué ocurrirá cuando se encuentren los dos escenarios: el de la formación inicial adquirida por el docente y el de la realidad en las escuelas. Como forma de anticipación de ese encuentro pedimos a un grupo de estudiantes de México y de España que valoren cuál creen ellos que es la situación en las escuelas en relación con la inclusión. De esa manera se ponen en juego los conocimientos que obtienen en la formación inicial sobre inclusión educativa, las competencias que adquieren y su capacidad de comprensión del contexto escolar. 


\section{MÉTODO}

La muestra de nuestro estudio está formada por estudiantes de Magisterio de México y de España. Concretamente pudimos contar con un grupo de 35 estudiantes de México y de 29 estudiantes de España.

El instrumento utilizado ha sido una selección de ítems del índice para la inclusión de Booth y Ainscow (2002). El instrumento se ha dividido en dos dimensiones: Crear culturas inclusivas y desarrollar prácticas inclusivas, que hemos considerado elementos claves para la inclusión en los centros educativos. Aunque el instrumento consta de 27 ítems, en este trabajo exponemos los resultados con los 6 primeros ítems, relacionados con la dimensión culturas inclusivas (construir comunidad). Son los siguientes:

1. Todo el mundo se siente acogido.

2. Los estudiantes se ayudan unos a otros.

3. El profesorado colabora entre sí.

4. Profesorado y alumnado se tratan con respeto.

5. Existe buena relación entre el profesorado y las familias.

6. Las instituciones de la localidad están involucradas en la escuela.

Los datos obtenidos con estos dos grupos de estudiantes han sido analizados con el paquete estadístico SPSS v 24.0., obteniéndose los siguientes resultados.

\section{RESULTADOS}

En este trabajo exponemos un adelanto de los resultados obtenidos con la dimensión primera sobre crear culturas inclusivas, y dentro de ella, la de construir una comunidad, ya que estos ítems constituyen elementos claves para la inclusión en el aula y en el centro.

Tabla 1: Construir una comunidad inclusiva en España.

\begin{tabular}{|c|c|c|c|c|c|c|}
\hline \multicolumn{7}{|c|}{ Estadísticos principales relacionados con la subdimensión: Construir una comunidad } \\
\hline & ACOGIDO & AYUDAN & PROF. COLABORAN & RESPETO & PROF-FAMILIAS & COMUNIDAD \\
\hline Media & 3,90 & 3,72 & 3,90 & 4,24 & 4,04 & 3,21 \\
\hline Mediana & 4,00 & 4,00 & 4,00 & 4,00 & 4,00 & 3,00 \\
\hline Moda & 3 & 4 & 4 & 5 & 5 & 3 \\
\hline Máximo & 5 & 5 & 5 & 5 & 5 & 5 \\
\hline Mínimo & 3 & 3 & 2 & 2 & 3 & 2 \\
\hline $\begin{array}{c}\text { Desviación } \\
\text { estándar }\end{array}$ &, 900 &, 591 &, 860 &, 830 &, 838 &, 884 \\
\hline
\end{tabular}

Podemos considerar el primero como el más importante, ya que puede aglutinar en cierto modo los demás. Podemos observar cómo puede considerarse que se cumple bastante, obteniéndose una media de casi 4 . Esto significa que en opinión de los estudiantes de magisterio español en general, en las escuelas, todo el mundo se siente tenido en cuenta, se siente valorado y acogido, aunque aún debe mejorarse algo.

El segundo indicador que podemos destacar como más importante es el de la cuarta columna: RESPETO, cuyo texto completo es: «Profesorado y alumnado se tratan con respeto». En este caso la puntuación es aún más alta, obteniéndose una media de 4,24. Esto quiere decir que lo que observan es que entre profesorado y alumnado en las escuelas el respeto es buenísimo, casi perfecto, lo que contradice en cierta medida lo que en ocasiones trasciende a los medios de comunicación sobre la pérdida de respeto hacia el profesorado. 
En tercer lugar de importancia podemos señalar la columna tercera denominada: AYUDAN, cuyo texto completo es "Los estudiantes se ayudan unos a otros». También en este caso, comoocurría en «todo el mundo se siente acogido», los resultados indican que se cumple bastante, con una media de 3,72. Se trata de una puntuación de casi 4 . Consideramos que se debe mejorar aún este aspecto para hacer mejores escuelas inclusivas.

Se trata, en general, de una dimensión bastante bien considerada, obteniéndose una media de medias de 3,8, lo que la sitúa muy cerca del 4:»se cumple bastante». Esto significa que el primer paso que necesitamos para crear escuelas inclusivas, referido a construir una comunidad acogedora, segura, que dé confianza y permita a todos sus miembros sentirse valorados está en buena situación, aunque se necesitará mejorar un poco más.

En el caso de México obtenemos los resultados siguientes:

Tabla 2: Construir una comunidad inclusiva en México.

\begin{tabular}{|c|c|c|c|c|c|c|}
\hline & ACOGIDO & AYUDAN & PROF. COLABORAN & RESPETO & PROFAMILIAS & COMUNIDAD \\
\hline Media & 2,41 & 2,74 & 2,94 & 3,23 & 2,42 & 2,06 \\
\hline Mediana & 3,00 & 3,00 & 3,00 & 3,00 & 2,00 & 2,00 \\
\hline Moda & 3 & 3 & 3 & 3 & 2 & 2 \\
\hline Máximo & 3 & 4 & 5 & 5 & 4 & 4 \\
\hline Mínimo & 1 & 1 & 2 & 1 & 1 & 1 \\
\hline $\begin{array}{c}\text { Desviación } \\
\text { estándar }\end{array}$ & ,743 & ,780 & ,906 & 1,031 & ,902 & ,851 \\
\hline
\end{tabular}

En este caso el valor de «Se siente acogido» es de 2,41, por lo que se encuentra más cerca de «Se cumple un poco». Los ítems que más alto puntúan son «los profesores colaboran entre sí», lo que puede ser un valor a tener en cuenta en el caso de México, y el respeto que se tienen profesorado y alumnado. En ambos casos se cumple aceptablemente. Siendo los ítems peor puntuados, los referidos a la relación profesorado-familias, con 2,42 y la involucración de las instituciones en la escuela, con un 2,06. Siendo esta la más baja.

La media de medias es de 2,63, que podemos considerar una puntuación baja, que se encuentra entre «se cumple un poco» y «Se cumple aceptablemente».

\section{DISCUSIÓN}

Justamente comparando las dos medias obtenidas con las medias de la dimensión analizada en ambos países obtenemos una visión inferior en México, en comparación con España, como puede apreciarse en la siguiente figura.

\section{Diferencia en cultura inclusiva}

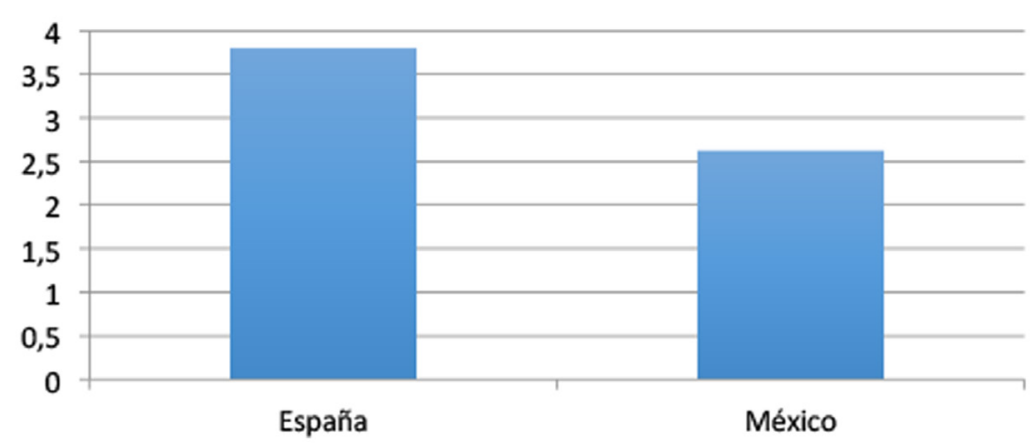

Figura 2. Comparación de las puntuaciones sobre cultura inclusiva (construir comunidad).

Haciendo la comparación entre las medias de los 6 indicadores estudiados en la dimensión cultura inclusiva (construir comunidad), hemos obtenido una puntación mayor en el caso español, aunque pensamos que en ambos casos debe ser rmejorada. 


\section{CONCLUSIONES}

- Se aprecia una distancia entre las leyes y normas sobre inclusión y equidad con respecto a la práctica cotidiana en los centros educativos. En nuestro trabajo se pone de manifiesto que hay legislación adecuada en ambos países pero las percepciones de los futuros docentes, que en gran medida coinciden con las de la ciudadanía se sitúan en un nivel entre bajo y medio.

- Una formación de docentes enfocada en los ítems que han tenido una puntuación más baja puede ser útil para mejorar la educación inclusiva.

- Es necesario profundizar en las respuestas educativas contextualizadas en los centros educativos de ambos países. Sólo con un sistema de prevención, detección, identificación, atención de necesidades, supervisión y vigilancia de su cumplimiento, es posible avanzar hacia una mejor educación inclusiva y por lo tanto justicia social. Más aún cuando la investigación pone de manifiesto la necesidad de impulsos continuos.

\section{REFERENCIAS BIBLIOGRÁFICAS}

Agencia Europea para el Desarrollo de la Educación del Alumnado con Necesidades Educativas Especiales, (2012) Perfil profesional del docente en la educación inclusiva Odense, Dinamarca: Agencia Europea para el Desarrollo de la Educación del Alumnado con Necesidades Educativas Especiales.

Alcántara, A. (2008). «Exclusión e inclusión en la educación superior: el caso de las universidades culturales en México», Inter-Ação (Brasil, Universidad Federal de Goias), vol. 33, núm. 1, enero-junio, pp. 151-167.

Alcántara, A. y Navarrete, Z. (2013). Inclusión Equidad y cohesión Social en las Políticas de Educación Superior en México. En RMIE, 2014, Vol. 19, Núm. 60, pp. 213-239.

Bolívar, A. (2011). Justicia social y equidad escolar. una revisión actual. En Revista Internacional de Educación para la Justicia social. (RIEJS). Vol. 1, Núm. 1, 2012, pp. 9-45.

Booth, T. y Ainscow, M. (2002). Guía para la evaluación y mejora de la educación inclusiva. Index for inclusión. Madrid, España: Consorcio Universitario para la educación inclusiva.

Confederación Española de Organizaciones en favor de las Personas con Discapacidad Intelectual - FEAPS (2009). La educación que queremos. Situación actual de la inclusión educativa en España. Madrid, España: FEAPS.

Dubet, F. (2011). Repensar la justicia social. Buenos Aires, Argentina: Siglo XXI.

Fernández, A. (2014). Inclusión y equidad. Una educación que multiplica oportunidades . Madrid, España: Entreculturas.

Instrucciones de 8 de marzo de 2017, de la Dirección General de Participación y Equidad, por las que se actualiza el protocolo de detección, identificación del alumnado con necesidades específicas de apoyo educativo y organización de la respuesta educativa.

Navarrete, Z. y Alcántara, A. (2013). La participación de los organismos internacionales en la promoción de políticas encaminadas a incluir y atender a estudiantes indígenas en la educación superior, en Navarro, M. A. y Navarrete, Z. (coords.) Comparar en educación. Diversidad de intereses, diversidad de enfoques, México: WCCES-SOMEC-COLTAM, pp.95-111.

Secretaría de Educación Pública (SEP) (2014). Programa para la Inclusión y la Equidad Educativa (PIEE). México: SEP. Recuperado de (https://inclusionyequidad.sep.gob.mx), el 4 de mayo de2018.

SEP (2012). Programa Atención a la Diversidad de la Licenciatura en Educación Primaria. México: SEP

Tedesco, J. C. (2012). Educación y justicia social en América Latina. Buenos Aires, Argentina: Fondo de Cultura Económica y Universidad Nacional de San Martín.

UNESCO (2009). Directrices sobre políticas de inclusión en la Educación. Paris, Francia: UNESCO.

UNESCO (2015). Declaración de Incheon y Marco de Acción para la realización del Objetivo de Desarrollo Sostenible 4. París, Francia: UNESCO.

Zacarías, J., De la Peña, A y Saad, E. (2006). Inclusión Educativa. México: S. M. 